\title{
Effectiveness of Corona Lockdowns: Evidence for a Number of Countries
}

\author{
Stefan Homburg \\ Discussion Paper No. 671 \\ ISSN 0949-9962
}

13. April 2020

Accepted: The Economist's Voice (forthcoming)

School of Economics and Management, Leibniz University of Hannover, Germany. Phone: +49 511 762-5633. Email: homburg@fiwi.uni-hannover.de.

Abstract

The paper assesses the effectiveness of the large-scale lockdowns that took place during the SARS-CoV-2 (corona) pandemic. Countries considered include the United States, South Korea, Germany, Austria, Switzerland, Italy, Spain, and Sweden. Our research strategy utilizes the fact that fatal outcomes follow infections with a delay of 23 days. Therefore, the dates of the actual infections can be inferred from the data. The results suggest that lockdowns were superfluous and ineffective.

JEL Classification: I18.

Keywords: Corona, SARS-CoV-2, COVID-19, Lockdown.

I would like to thank the editor of The Economist's Voice and two anonymous referees, who spent a weekend to speed up the process and who provided very useful hints. 


\section{Introduction}

During the corona pandemic, reported diagnoses (referred to as "total cases" in the Johns Hopkins database) found great interest. Now, after the pandemic has receded in many countries, it appears preferable to use "total deaths" instead. This is because test frequencies vary considerably over time and between countries. Moreover, one can expect that deaths, which were much smaller in number than diagnoses, are documented with greater care. It is known, however, that many countries count all fatal outcomes with a positive diagnose as "corona death", i.e., irrespective of the genuine cause. This inflates the figures.

Pandemics are usually described by logistic functions. These are also referred to as epidemiological functions, cf. Brauer, F. and C. Castillo-Chavez (2001). They are used to describe infections, positive diagnoses, or fatal outcomes and can be employed in the analysis of the corona pandemic, as will be seen immediately.

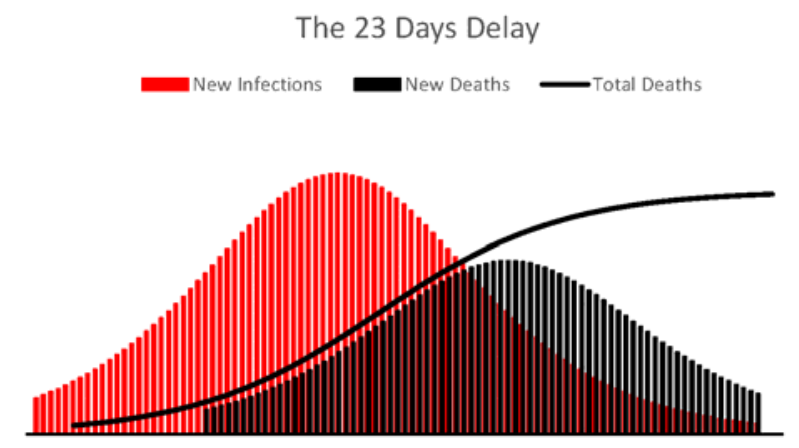

Figure 1: Infections, deaths, and the logistic function.

In figure 1, the black line depicts total deaths, which follow a logistic function. The black bars represent the function's density, i.e. daily new deaths, and the red bars correspond to the actual infections, which are not directly observable. To repeat, "new cases", or diagnoses, are not used here.

The medical literature reports a mean incubation period of 5 days (Lauer et al. 2020) and a mean period of 18 days from onset of symptoms to death (Verity et al. 2020, cf. also Sanche et al. 2020). Combining the two numbers yields a lag between infection and death of 23 days. In figure 1, this lag of 23 days corresponds to the horizontal distance between the two maxima. Note that infections are very much higher than fatal outcomes, so the two vertical axes are scaled differently. The problem that the total number of infections is unknown does not affect the following analysis because the latter employs only the lag between the two distributions.

\section{Descriptive Statistics}

The paper considers a number of industrialized countries over the period 01 March 2020 until 13 April. The starting point was chosen because most countries did not report fatal 
outcomes earlier. The end point is the day of publication. All data were taken from the Johns Hopkins database ${ }^{1}$. Logistic functions take the following form:

$$
f(t)=\frac{S}{1+a e^{-b t}} .
$$

Here, $t$ denotes time, and $S$ represents the saturation limit to which the values converge. The logistic function solves the differential equation $f^{\prime}(t)=f(t)[S-f(t)]$ that has a nice intuition: New infections grow linearly in the number of persons already infected, $f(t)$, and grow also linearly in the number of persons that are susceptible to the infection but not yet infected, the term $S-f(t)$. As infections approach $S$, new infections converge to zero. With a given case fatality rate, fatal outcomes follow the same path with a delay of 23 days and a much smaller incidence. This paper utilizes only the delay but not the case fatality rate, which is highly uncertain.

Table 1 presents results for Austria (A), Switzerland (CH), Germany (D), Spain (E), Italy (I), South Korea (RSK), Sweden (S), the United Kingdom (UK), and the United States (US). The parameter estimates were inferred from a non-linear time series analysis.

The second column predicts the mortalities at end of the pandemic. Each mortality was calculated as saturation limit over total population. Final mortalities will be relatively high in Spain and Italy, and relatively low in Austria, Germany, and South Korea. Of course, excess mortalities will likely be lower, but reliable data are not available at this point.

$\begin{array}{cccc}\text { Country } & \text { Mortality \% } & \text { Turning point } & \mathbf{R}^{\mathbf{2}} \\ \text { A } & 0.005 \% & \text { April 6 } & 0.996 \\ \text { CH } & 0.015 \% & \text { April 04 } & 0.999 \\ \text { D } & 0.005 \% & \text { April 7 } & 0.999 \\ \text { E } & 0.039 \% & \text { April 1 } & 0.998 \\ \text { I } & 0.035 \% & \text { March 29 } & 0.999 \\ \text { RSK } & 0.001 \% & \text { March 26 } & 0.995 \\ \text { S } & 0.012 \% & \text { April 8 } & 0.999 \\ \text { UK } & 0.023 \% & \text { April 9 } & 1.000 \\ \text { US } & 0.010 \% & \text { April 10 } & 0.997\end{array}$

Table 1: Descriptive statistics.

The figures in the third column are the turning points of the respective logistic functions, or the maxima of their densities. From the symmetry of the density function, it follows that the position of a turning point is implicitly defined by the equation $f(t)=S / 2$. Solving yields $t=-\ln (1 / a) / b$ so that the position follows directly from the estimated parameters. Corona infections receded early in South Korea, which was hit shortly after China, and also in Italy. The United Kingdom and the United States were affected relatively lately. The high coefficients of determination from the final column demonstrate that logistic functions approximate the data with high accuracy.

1 https://github.com/CSSEGISandData/COVID-19/blob/master/csse_covid_19_data/csse_covid_19_time_series/time_series_covid19_deaths_global.csv 


\section{Timing and Impact of the Lockdowns}

Except South Korea and Sweden, all countries under consideration locked down their economies for weeks. These extreme measures induced large economic costs. The results shown in table 2 examine the timing of the lockdown policies and suggest answers to the question whether or not the lockdowns were necessary.

The data in table 1 above already provide a first hint: Mortalities in South Korea and Sweden are inconspicuous. South Korea has the lowest mortality of all countries, and Sweden's mortality takes an intermediate value. Of course, South Korea employed aggressive tracing, but Sweden did not. Both countries kept their bars, schools and shops open during the entire crisis but did not suffer from "exponential growth" of any kind. The following observations may help explaining these spurious anomalies.

$\begin{array}{ccc}\text { Country } & \text { Turning point-23 } & \text { Lockdown } \\ \text { A } & \text { March 14 } & \text { March 16 } \\ \text { CH } & \text { March 12 } & \text { March 16 } \\ \text { D } & \text { March 15 } & \text { March 23 } \\ \text { E } & \text { March 9 } & \text { March 13 } \\ \text { I } & \text { March 6 } & \text { March 11 } \\ \text { RSK } & \text { March 3 } & \text { none } \\ \text { S } & \text { March 16 } & \text { none } \\ \text { UK } & \text { March 17 } & \text { March 23 } \\ \text { US } & \text { March 18 } & \text { March 21 }\end{array}$

Table 2: Timing of Lockdown Policies.

The dates in the second column were obtained by subtracting 23 from the dates in the third column in table 1 . Following the medical literature cited above, the unobservable infections reached their respective maxima at these dates, when infections started receding. Interestingly, all countries that used lockdowns implemented them after the turning points, when the bulk of infections had already taken place. South Korea and Sweden seem to be the only countries in the sample which accounted for the established fact that every pandemic follows a logistic, rather than an exponential, growth path. 


\section{Italy as an Illustration}

Outside China's Hubei province, the first lockdown took place in Italy. Hence, this country can be taken as a model case that deserves closer inspection. Figure 2 shows actual daily fatalities from the Johns Hopkins database as blue and green bars. The red line represents the density of the estimated logistic function.

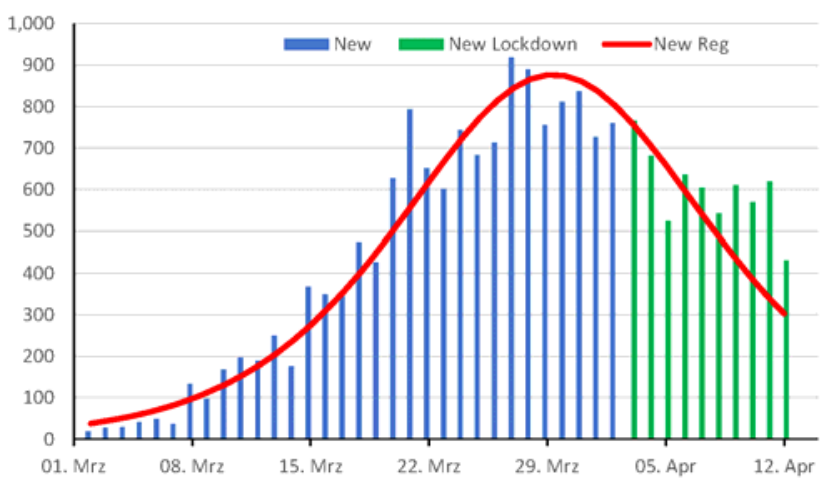

Figure 2: Italy's Death Rates before and after the Lockdown.

In principle, the fatal outcomes indicated by green (shaded) bars on the right could have been influenced by the lockdown because the lockdown became effective 11 March, and the green bars start on 2 April, 23 days after the lockdown. By contrast, the delay between true infections and fatalities rules out that the blue bars were affected by the lockdown. Obviously, the lockdown had no visible impact on fatal outcomes.

The main takeaway from figure 2 is that Italy implemented the lockdown too late. The lockdown became effective when infections were already receding and roughly after two thirds of total infections with fatal outcomes had already been realized. Therefore, the measure was neither necessary nor effective.

\section{Conclusion}

This paper has demonstrated that countries with lockdowns did not perform better with respect to fatal outcomes, if compared with countries that refused lockdowns. Its main purpose, however, is a methodical suggestion. Observed "total cases", or positive diagnoses, are highly unreliable data since they depend on testing habits and numbers. Total deaths, by contrast, are much more reliable. Making use of medical evidence, according to which deaths follow true infections with an average delay of 23 days, the paper proposes that in order to describe the pandemic's dynamics, it is preferable to use total deaths and the delay, rather than total cases.

In order to avoid any misunderstanding, the paper's message does not state that doing nothing had been optimal. Traditional mitigation strategies such as testing, tracing, social distancing, and quarantining are very important; there can be no doubt that these measures reduced the saturation levels of infections and fatal outcomes. The same may hold true with respect to announcement effects that require future research. The paper questions, however, that the lockdowns of entire economies yielded additional benefits. 


\section{References}

Brauer, F. and C. Castillo-Chavez (2001) Mathematical Model in Population Biology and Epidemiology. Springer.

Lauer, S. A. et al. (2020) The Incubation Period of Coronavirus Disease 2019 (COVID19) from Publicly Reported Confirmed Cases: Estimation and Application. Annals of Internal Medicine, https://doi.org/10.7326/M20-0504.

Sanche, S. et al. (2020) High Contagiousness and Rapid Spread of Severe Acute Respiratory Syndrome Coronavirus 2. Emerging Infectious Diseases 2020, https://doi.org/10.3201/eid2607.200282.

Verity, R. et al. (2020) Estimates of the Severity of Coronavirus Disease 2019: A Modelbased Analysis. Lancet 30 March 2020, https://doi.org/10.1016/S1473-3099(20)30243-7.

* I would like to thank the editor and two anonymous referees, who spent a weekend to speed up the process and provided very useful hints. 